\title{
Gastric duplication cyst-a case report
}

\begin{abstract}
Commonest site for enteric duplications is small bowel and it is extremely uncommon for gastroduodenal region to get affected. Presentation maybe antenatal on anomaly scan or post natally when symptomatic due to complications. We report a 5month old girl who was brought with malena, abdominal pain and vomiting of two days duration with antenatal Ultrasound showing a cystic mass of unknown origin. On exploration, mass in the gastroduodenal region was found. Wide local excision with a part of the gastric wall \&tail of pancreas was done. Histopathology was suggestive of gastric duplication cyst.
\end{abstract}

Keywords: gastric duplication, enteric duplication
Volume 5 Issue 6 - 2016

\section{Pradnya S Bendre,Amol Nage, Flavia D'souza,} Nitin palse

Department of paediatric surgery, Baijerbai Wadia Hospital for children, India

Correspondence: Pradnya Bendre, Department of paediatric surgery, B JWadia Children's hospital, for children, Acharya Dhonde Marg Parel-4000I2 Mumbai, India, Tel +919324567237, Email suhaspradnya@yahoo.com

Received: July 31, 2016 | Published: November 30, 2016

\section{Introduction}

Among all enteric duplications, the ones occurring in the gastroduodenal region are rare accounting for only seven percent of all duplications..$^{1-3}$ We report an interesting presentation as acute abdomen which turned out to be because of gastric duplication cyst.

\section{Case report}

5 month old girl presented in emergency with malena, abdominal pain and non bilious, non- projectile vomiting of two days duration. Antenatal sonography at 33 wks of gestation had shown cystic lesion of $37 \times 27 \times 26 \mathrm{~mm}$. On admission child was febrile. Abdominal examination revealed tenderness and guarding. X-ray abdomen was unremarkable. Ultrasound showed a large heterogenous hypoechoic lesion $4.4 \times 3.5 \mathrm{~cm}$ just distal to splenic anterior pole, walled off by greater omentum. CT scan showed heterogenous hypoechoic lesion in the region of stomach of $4 \mathrm{~cm} \times 5 \mathrm{~cm}$ size in the left hypochondrium and epigatric region (Figures 1\&2). After initial stabilization, child was explored. There was a mass behind stomach adherent to transverse colon and tail of pancreas. Colon was dissected from stomach. It was difficult to differentiate whether it was neoplastic or not. Wide local resection of gastric mass along with part of wall \&terminal pancreas was done. Stomach wall was closed in layers. Feeding jejunostomy tube was placed $15 \mathrm{~cm}$ away from DJ junction. Specimen was sent for histopathological examination (Figure $3 \& 4$ ). Post-operative recovery was uneventful. Histopathological examination revealed gastric mucosa and smooth muscles in the wall of cyst which confirmed the diagnosis of gastric duplication cyst. At six months follow up patient is asymptomatic and thriving well.

\section{Discussion}

Gastro-duodenal duplications are rare $^{1,2}$ and account for approximately seven per cent of all gastrointestinal duplication cysts. ${ }^{3}$ In the era of anomaly scan most of the cysts are detected antenatally, however locating organ of origin may be difficult .Symptomatic presentation depends on the size, position ${ }^{3}$ and presence or absence of complications and include gastric outlet obstruction, abdominal pain or an abdominal mass. Embryo pathogenesis of enteric duplications cannot explain occurrence at gatroduodenal region as well as none of the theories quoted in literature have solved the mystery.,3

Histopathological criteria for diagnosis of gastric duplication cyst include the wall of the cyst being contiguous with the stomach wall, the presence of smooth muscle surrounding the cyst and in continuation with the gastric musculature, and lining of the cyst wall by epithelial, gastric, or gut mucosa of any type..$^{4-8}$

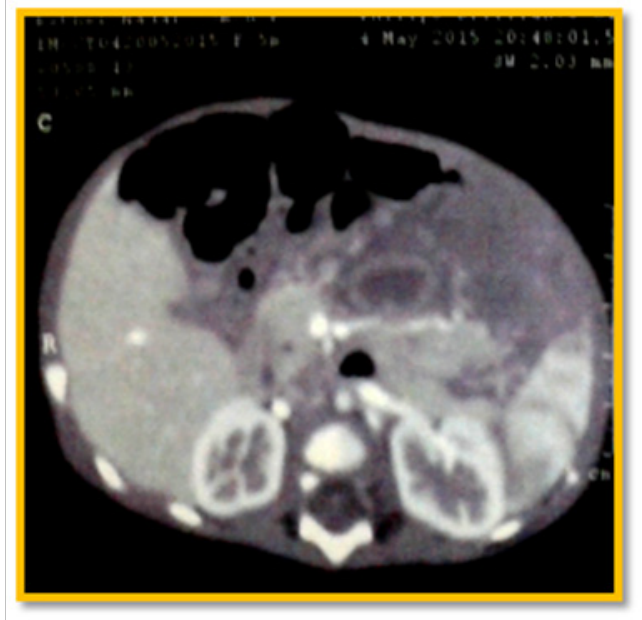

Figure I CT scan image.

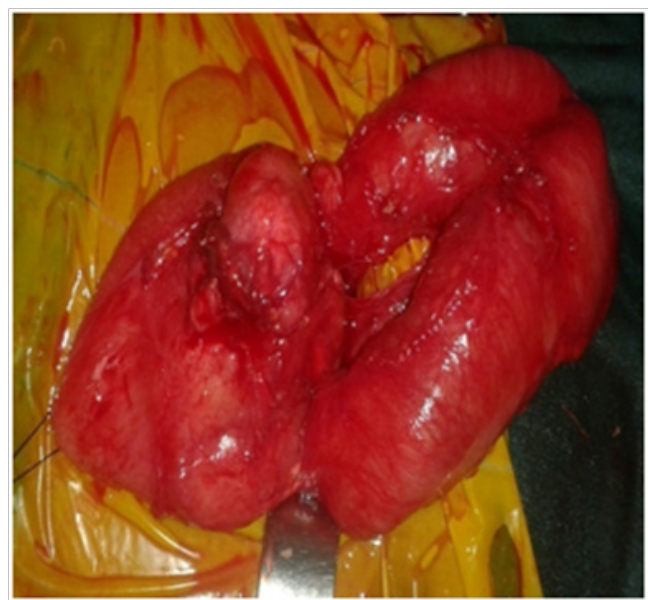

Figure 2 Intraop Gastric mass.

CT imaging can precisely define location, size, and local tissue involvement. Typical appearance is that of cystic lesions with thick walls which often show contrast enhancement of the inner lining. Endoscopic ultrasound shows cystic lesion with hypoechoic muscle layer and echogenic internal mucosal layer. ${ }^{9}$ 


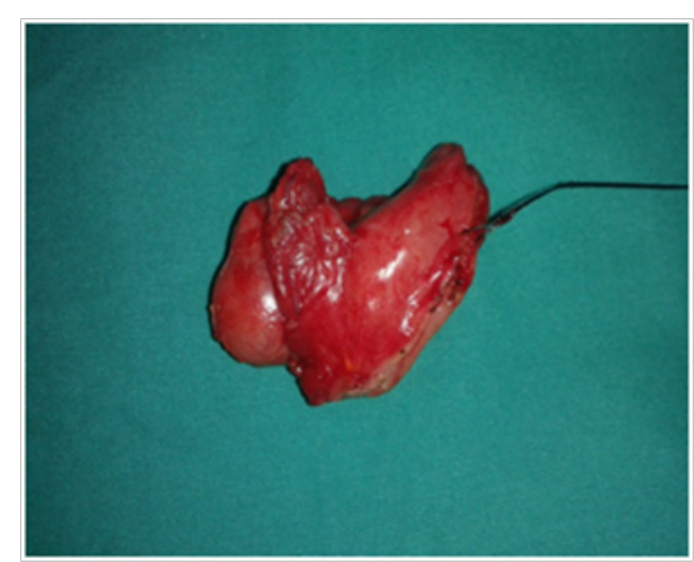

Figure 3 Specimen.

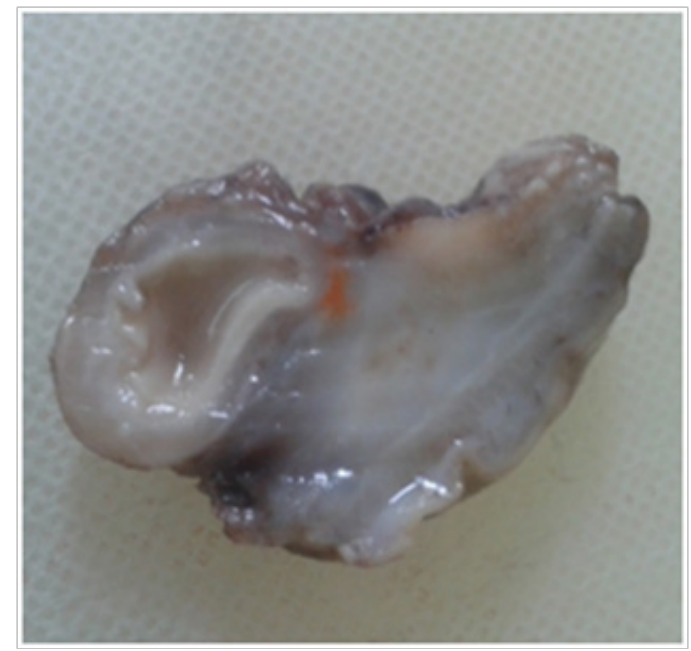

Figure 4 Cut section of specimen.

Complete excision is treatment of choice. Potential of these cysts to adhere to surrounding structures makes surgery intricate. Differentiation from neoplasia is also difficult intra-operatively. Marsupialization and stripping of the mucosal lining is the second option when it is not possible to find a plane between the cyst and the stomach. There are few cases in the literature successfully treated by laparoscopic resection. ${ }^{10}$ Because of the potential for neoplastic transformation, it is recommended that duplication cysts be surgically excised. ${ }^{11}$

\section{Conclusion}

Although rare, gastrointestinal duplication cysts are unique entities that are often detected after significant growth with development of compressive symptoms. They are often diagnosed postoperatively by careful pathologic examination, and treatment of choice remains complete surgical resection.

\section{Acknowledgments}

None.

\section{Conflicts of interest}

Authors declare that there is no conflict of interest.

\section{References}

1. Macpherson RI. Gastrointestinal tract duplications: Clinical, pathologic, etiologic, and radiologic considerations. Radiographics. 1993;13(5):1063-1080.

2. Lee NK, Kim S, Jeon TY, et al. Complications of congenital and developmental abnormalities of the gastrointestinal tract in adolescents and adults: Evaluation with multimodality imaging. Radiographics. 2010;30(6):1489-1507.

3. Berrocal T, Torres I, Gutiérrez J, et al. Congenital anomalies of the upper gastrointestinal tract. Radiographics. 1999;19(4):855-872.

4. Kuraoka K, Nakayama H, Kagawa T, et al. Adenocarcinoma arising from a gastric duplication cyst with invasion to the stomach: a case report with literature review. Journal of Clinical Pathology. 2004;57(4):428-431.

5. Singh JP, Rajdeo H, Bhuta K, et al. Gastric duplication cyst: two case reports and review of the literature. Case Reports in Surgery. 2013;2013:4.

6. Johnston J, Wheatley GH, El Sayed HF, et al. Gastric duplication cysts expressing carcinoembryonic antigen mimicking cystic pancreatic neoplasms in two adults. The American Surgeon. 2008;74(1):91-94.

7. Horne G, Ming-Lum C, Kirkpatrick AW, et al. High-grade neuroendocrine carcinoma arising in a gastric duplication cyst: a case report with literature review. International Journal of Surgical Pathology. 2007;15(2):187-191.

8. Mardi K, Kaushal V, Gupta S. Foregut duplication cysts of stomach masquerading as leiomyoma. Indian J Pathol Microbiol. 2010;53(1):160-161.

9. Maeda H, Okabayashi T, Nishimori I, et al. Diagnostic challenge to distinguish gastric duplication cyst from pancreatic cystic lesions in adult. Inter Med. 2007;46(14):1101-1104.

10. Ford WD, Guelfand M, López PJ, et al. Laparoscopic excision of a gastric duplication cyst detected on antenatal ultrasound scan. J Pediatr Surg. 2004;39(10):e8-e10.

11. Johnston J, Wheatley GH, El Sayed HF, et al. Gastric duplication cysts expressing carcinoembryonic antigen mimicking cystic pancreatic neoplasms in two adults. Am Surg. 2008;74(1):91-94. 